\title{
QT Variability Index is Correlated with Autonomic Nerve Activity in Healthy Children
}

\author{
Hirofumi Kusuki ${ }^{1} \cdot$ Yuka Tsuchiya $^{1} \cdot$ Yuri Mizutani $^{1} \cdot$ Miki Nishio $^{1} \cdot$ Shota Oikawa $^{2} \cdot$ Rina Nagata $^{2} \cdot$ Yumi Kiriyanagi $^{2}$. \\ Kayo Horio ${ }^{3}$. Arisa Kojima ${ }^{4} \cdot$ Hidetoshi Uchida $^{4} \cdot$ Namiko Kojima $^{5} \cdot$ Kazuyoshi Saito $^{5} \cdot$ Tsuneaki Sadanaga $^{6}$. \\ Tadayoshi Hata ${ }^{1}$ (1)
}

Received: 4 March 2020 / Accepted: 12 June 2020 / Published online: 22 June 2020

(c) The Author(s) 2020

\begin{abstract}
The QT variability index (QTVI), which measures the instability of myocardial repolarization, is usually calculated from a single electrocardiogram (ECG) recording and can be easily applied in children. It is well known that frequency analysis of heart rate variability (HRV) can detect autonomic balance, but it is not clear whether QTVI is correlated with autonomic tone. Therefore, we evaluated the association between QTVI and HRV to elucidate whether QTVI is correlated with autonomic nerve activity. Apparently, healthy 320 children aged 0-7 years who visited Fujita Health University Hospital for heart checkup examinations were included. The RR and QT intervals of 60 continuous heart beats were measured, and the QTVI was calculated using the formula of Berger et al. Frequency analysis of HRV, including the QTVI analysis region, was conducted for $2 \mathrm{~min}$ and the ratio of low-frequency (LF) components to high-frequency (HF) components (LF/HF) and $\mathrm{HF} /(\mathrm{LF}+\mathrm{HF})$ ratio was calculated as indicators of autonomic nerve activity. Then, the correlations between QTVI and these parameters were assessed. QTVI showed a significant positive correlation with $\mathrm{LF} / \mathrm{HF}$ ratio $(r=0.45, p<0.001)$ and negative correlation with $\mathrm{HF} /(\mathrm{LF}+\mathrm{HF})$ ratio $(r=-0.429, p<0.001)$. These correlations remained after adjustment for sex and age. QTVI, which is calculated from non-invasive ECG and can detect abnormal myocardial repolarization, is significantly correlated with frequency analysis of HRV parameters. QTVI reflects autonomic nerve balance in children.
\end{abstract}

Keywords QT variability index $\cdot$ Heart rate variability $\cdot$ Prepubescent $\cdot \mathrm{LF} / \mathrm{HF} \cdot \mathrm{HF} /(\mathrm{LF}+\mathrm{HF})$

Tadayoshi Hata

thata@fujita-hu.ac.jp

1 Graduate School of Health Sciences, Fujita Health University, Toyoake, Aichi, Japan

2 Laboratory of Clinical Medicine, Fujita Health University Hospital, Toyoake, Aichi, Japan

3 Department of Physiology, School of Medicine, Fujita Health University, Toyoake, Aichi, Japan

4 Department of Pediatrics, School of Medicine, Fujita Health University, Toyoake, Aichi, Japan

5 Department of Pediatrics, Meijyo Hospital, Nagoya, Aichi, Japan

6 Seigato Hospital, Kumamoto, Japan

\section{Introduction}

Instability of myocardial repolarization detected by the QT interval variability indicates a substrate that can induce lethal arrhythmias [1]. Adult patients with myocardial dysfunction exhibit high QT interval variability following the preceding cardiac cycle, and such increased QT variability may be an ominous sign of cardiac death [2]. On the other hand, heart rate variability (HRV) calculated from the variations in the RR interval reflects autonomic nerve balance. Reduced HRV can predict the poor prognosis in patients with heart diseases [3, 4]. However, there were limited studies assessing the myocardial repolarization in children, and sufficient clinical applications have not been achieved.

The changes in QT interval variability are linked to autonomic and central nervous system [5]. The QT Variability Index (QTVI) is a non-invasive measure to assess repolarization liability that has been applied to a wide variety of subjects with cardiovascular disease [6]. We have used QTVI 
to evaluate the characteristics of myocardial repolarization in infants. This index changes with age in healthy children from infancy to school age [7, 8]. In healthy 1-month-old infants, the QTVI is negatively correlated with gestational age, which can serve as an index of the maturity of the cardiac autonomic nervous system and myocardial repolarization [9]. In the present study, we examined the correlation between QTVI and the power spectral analysis parameters of HRV, which are commonly used to measure autonomic nerve balance.

\section{Participants and Methods}

Apparently healthy 320 children aged $0-7$ years who visited Fujita Health University Hospital for heart checkup examinations between April 2012 and November 2015 were included. For children aged younger than 1 year, tricloryl syrup $(0.7 \mathrm{~mL} / \mathrm{kg})$ was used for sedation to perform such diagnostic procedures. Informed consents were obtained from the children's parents or guardians.

ECG was performed using a bio-polygraph recorder (MP150; Biopac Systems Inc., CA, USA) with a sampling rate of $1000 \mathrm{~Hz}$, and signals of the CM5 lead were recorded. All ECG recordings were obtained between 4 PM and 6 PM.

The RR interval was automatically measured using AcqKnowledge version 3.9 (Biopac Systems Inc., CA, USA) on the basis of the ECG recordings with stable baseline. The endpoint of the $T$ wave was identified using the firstorder differentiation processing method (Fig. 1). For 60 heart beats, we calculated the instantaneous heart rate, as well as the mean HR (HRm), mean QT interval (QTm), and variance (HRv and QTv). Thereafter, using the formula of Berger et al., we calculated the QTVI [QTVI $=\log _{10}(\mathrm{QTV} /$ $\left.\left.\mathrm{QTm}{ }^{2}\right) /\left(\mathrm{HRv} / \mathrm{HRm}^{2}\right)\right]$, which is an indicator of variability in the QT interval.

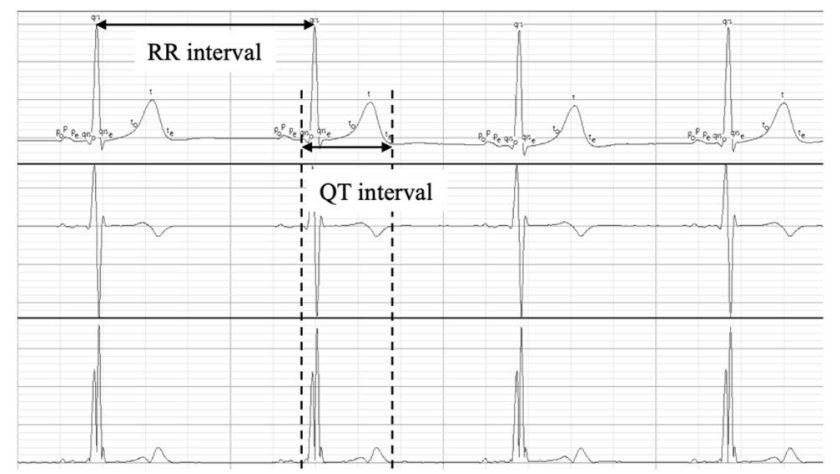

Fig. 1 Measurement of RR interval and QT interval. The ECGs were recorded by the CM5 lead using a Biopac biological polygraph recording device. $\mathrm{Q}$ onset, $\mathrm{T}$ end, and preceding $\mathrm{RR}$ intervals were measured using first derivative (b) and absolute functions (c) from 60 consecutive beats with a stable baseline ECG
Meanwhile, to evaluate the autonomic nerve function, we performed power spectrum analysis of HRV during a short period of 2 min including the QTVI analysis region using AcqKnowledge version 3.9. In power spectrum analysis, we measured the frequency density of the low-frequency region (LF: $0.036-0.146 \mathrm{~Hz}$ ) and the high-frequency region (HF: $0.146-0.390 \mathrm{~Hz}$ ) according to the method shown in The Task Force of the European Society of Cardiology and the North American Society of Pacing and Electrophysiology [10]. Then, LF/HF and $\mathrm{HF} /(\mathrm{LF}+\mathrm{HF})$ were calculated as indicators of autonomic nerve balance. Then, the correlations between QTVI and these parameters were assessed. The association of time domain HRV and QTVI was also assessed. In this study, standard deviation of NN intervals (SDNN) and root mean square of successive RR interval differences (rMSSD) were used. These values were expressed in original units or as the logarithm from $\left(\log _{10}\right)$ to obtain normal distribution.

\section{Statistical Analysis}

Statistical processing was performed using the JMP version 12.2.0 (SAS Institute Inc., Cary, NC, USA). The Wilcoxon signed rank test was used for Sex-specific comparison. Correlation between QTVI and age was assessed using logarithmic curve regression analysis. Correlations between QTVI and $\mathrm{LF} / \mathrm{HF}$ or $\mathrm{HF} /(\mathrm{LF}+\mathrm{HF})$ were assessed using Pearson's linear regression analysis. Additionally, correlations between QTVI and SDNN or rMSSD were assessed using logarithmic curve regression analysis.

Multiple regression analysis was performed to assess whether QTVI was associated with HRV parameters independent of age and sex. A $p$ value of $<0.05$ was accepted as statistically significant.

\section{Results}

\section{Participants' Characteristics, ECG Parameters and HRV Parameters}

Table 1 presents the characteristics of the participants. The median age was 3 years. Median QT interval was $318.6 \mathrm{~ms}$ with a median heart rate of 103.3 beats per minutes. The corrected QT intervals by Bazett's and Fridericia's formulas were 414.8 and $380.0 \mathrm{~ms}$, respectively. None of the children had a corrected QT interval exceeding $440 \mathrm{~ms}$. In the HRV parameters, median LF/HF ratio, reflecting sympathetic nerve activity, was 1.75 , whereas median $\mathrm{HF} /(\mathrm{LF}+\mathrm{HF})$, reflecting vagal nerve activity, was 0.36 . 
Table 1 Characteristics of the study population

\begin{tabular}{ll}
\hline Clinical characteristics & 320 \\
Number & $177 / 143$ \\
Male/Female & $42.5[16.0-64.0]$ \\
Age (months) & $3.0[1.0-5.0]$ \\
Age (years) & \\
HRV parameters & $1716[925-2893]$ \\
LF (ms $\left.{ }^{2}\right)$ & $964[443-2120]$ \\
HF $\left(\mathrm{ms}^{2}\right)$ & $1.75[0.89-3.48]$ \\
LF/HF & $0.36[0.22-0.53]$ \\
HF/(LF+HF) & \\
ECG parameters & $103.3[91.9-115.1]$ \\
HR (bpm) & $583.4[521.9-657.8]$ \\
RR (ms) & $318.6[294.4-336.5]$ \\
QT (ms) & $414.8[402.8-426.3]$ \\
QTcB (ms) & $380.0[364.5-392.1]$ \\
QTcF (ms) & $25.4[13.5-39.5]$ \\
HRv (bpm $\left.{ }^{2}\right)$ & $15.8[8.2-28.8]$ \\
QTv (ms $\left.{ }^{2}\right)$ & $-2.63[-2.95$ to -2.39$]$ \\
$\log _{10} \mathrm{HRVN} \log _{10}$ QTVN & $-3.80[-4.07$ to -3.51$]$ \\
QTVI & $1.23[-1.53$ to -0.80$]$ \\
\hline
\end{tabular}

Each value is expressed as median [interquartile range]. Comparisons between male and female were performed with the Wilcoxon signed rank test

$L F$ low frequency density, $H F$ high frequency density, $L F / H F$ the ratio of low-frequency components to high-frequency components, $H F /(L F+H F)$ the ratio of high-frequency components to low-frequency + high-frequency components, $H R$ heart rate, $R R$ RR interval, $Q T$ QT interval, $Q T c B$ corrected QT interval by Bazett's formula, $Q T c F$ corrected QT interval by Fridericia's formula, $H R V N$ normalized HR variance, $Q T V N$ normalized QT variance, $Q T V I \mathrm{QT}$ variability index, $\beta$ standard regression coefficient, SEM standard error of the mean

Table 2 Gender difference in QTVI and HRV parameters

\begin{tabular}{llll}
\hline & Male & Female & $P$ \\
\hline QTVI & $-1.23[-1.52$ to & $-1.25[-1.54$ to & 0.632 \\
& $-0.77]$ & $-0.86]$ & \\
$\log _{10}$ HRVN & $-2.64[-3.04$ to & $-2.59[-2.8754$ to & 0.531 \\
& $-2.37]$ & $-2.41]$ & \\
$\log _{10}$ QTVN & $-3.78[-4.05$ to & $-3.82[-4.0854$ to & 0.684 \\
& $-3.51]$ & $-3.52]$ & \\
\hline
\end{tabular}

\section{Gender Differences in QTVI and HRV Parameters}

There were no gender differences for the QTVI, $\log _{10}$ HRVN, and $\log _{10}$ QTVN (Table 2).
Relationships Between QTVI and Age, Between LF/ HF and Age

Figure 1 presents the relationship between QTVI and age and between $\mathrm{LF} / \mathrm{HF}$ and age. Both indices decreased rapidly up to 12 months of age and slowly decreased thereafter.

\section{Relationship Between QTVI and LF/HF, Between QTVI and HF/(LF + HF)}

Figure 2 presents the relationship between QTVI and LF/ HF and between QTVI and HF/(LF+HF). A significant positive correlation was observed between QTVI and LF/ HF ( $r=0.450, p<0.001)$, whereas a significant negative correlation was observed between QTVI and $\mathrm{HF} /(\mathrm{LF}+\mathrm{HF})$ $(r=-0.429, p<0.001)$. A significant correlation between $\log _{10} \mathrm{HRVN}$ and $\mathrm{LF} / \mathrm{HF}, \mathrm{HF} /(\mathrm{LF}+\mathrm{HF})$ were observed ( $r=-0.415, p<0.001, r=0.386, p<0.001$, respectively). Similar correlation between $\log _{10} \mathrm{QTVN}$ and LF/HF, HF/ (LF+HF) were observed $(r=0.144, p=0.010, r=-0.151$, $p=0.007$, respectively), but the correlations were weak (Fig. 3).

\section{Relationship Between QTVI and SDNN, Between QTVI and rMSSD}

A significant correlation between QTVI and $\log _{10} \mathrm{SDNN}$ and $\log _{10} \mathrm{rMSSD}$ was observed $(r=-0.784, p<0.001, r=0.756$, $p<0.001$, respectively).

\section{Multivariate Analysis}

Table 3 shows multiple regression analysis. QTVI was significantly correlated with $\mathrm{LF} / \mathrm{HF}$ and $\mathrm{HF} /(\mathrm{LF}+\mathrm{HF})$ after adjustment for age and sex.

\section{Discussion}

The present study demonstrated that the QTVI is correlated with autonomic nerve activity in prepubescent healthy children aged 0-7 years. Namely, QTVI showed a significant positive correlation with $\mathrm{LF} / \mathrm{HF}$ ratio reflecting sympathetic nerve activity and negative correlation with $\mathrm{HF} / \mathrm{LF}$ $+\mathrm{HF}$ ) ratio, reflecting vagal nerve activity. QTVI showed a significant negative correlation with SDNN reflecting the sympatho-vagal nervous balance and negative correlation with rMSSD, reflecting vagal nerve activity.

Berger et al. [2] proposed that temporal variations in the QT interval-instability in myocardial repolarizationcould serve as an electrophysiological indicator. QT interval is affected by the heart rate; larger HRV could induce larger QT interval variability, whereas smaller HRV could 

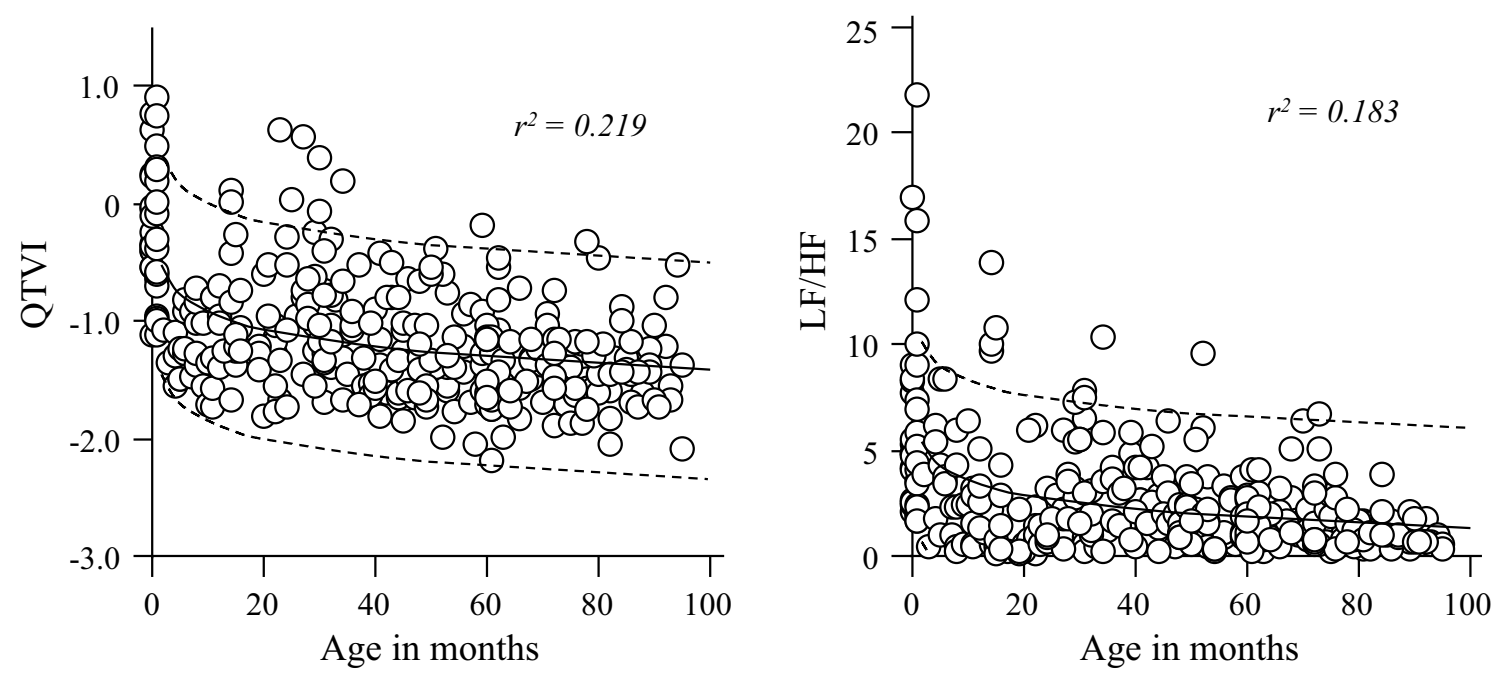

Fig. 2 Relationships between QTVI or LF/HF and age in months. QTVI and LF/HF decreased rapidly until 12 months after birth, following which it gradually decreased during infancy and thereafter became constant when they reached preschool age

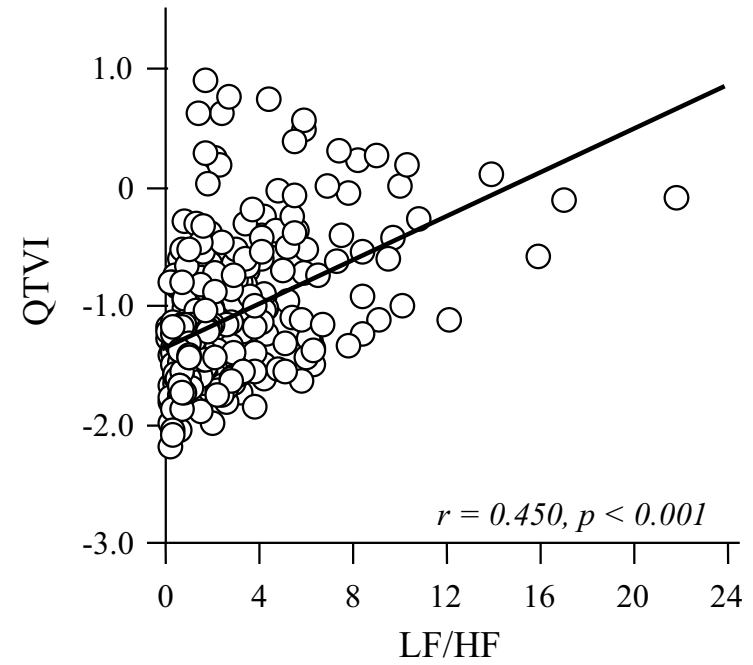

Fig. 3 Relationships between QTVI and HRV parameters and QTVI and $\mathrm{LF} / \mathrm{HF}$ show a positive correlation $(r=0.450, p<0.001)$. QTVI and $\mathrm{HF} /(\mathrm{LF}+\mathrm{HF})$ show a significant negative correlation $(r=-0.429, p<0.001)$. $L F / H F$ the ratio of low-frequency compo-

Table 3 The multiple regression analysis with QTVI as age, sex, and HRV parameters

\begin{tabular}{lrlr}
\hline Independent variable & \multicolumn{1}{l}{$P$} & SEM & \multicolumn{1}{l}{$P$} \\
\hline Age (years) & -0.291 & 0.044 & $<0.001$ \\
Sex & 0.019 & 0.054 & 0.723 \\
LF/HF & 0.711 & 0.113 & $<0.001$ \\
Age (years) & -0.313 & 0.043 & $<0.001$ \\
Sex & 0.038 & 0.054 & 0.486 \\
HF/(LF+HF) & -0.400 & 0.062 & $<0.001$ \\
\hline
\end{tabular}

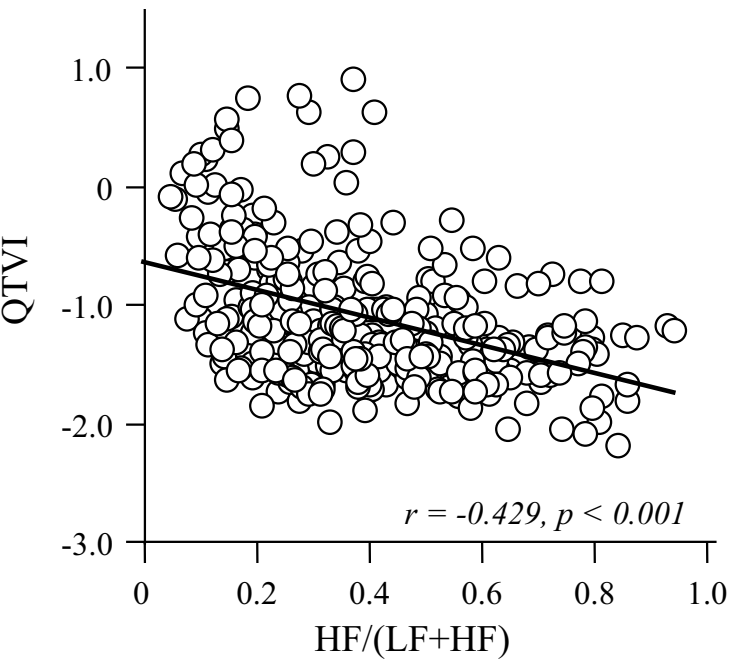

nents to high-frequency components, $H F /(L F+H F)$ the ratio of highfrequency components to low-frequency +high-frequency components

induce smaller QT interval variability. Either overestimation or underestimation of QT variability might occur without considering the degree of HRV. Therefore, the authors proposed the formula: QTVI $=\log _{10}\left(\mathrm{QTv} / \mathrm{QTm}{ }^{2}\right) /(\mathrm{HRv} /$ $\mathrm{HRm}^{2}$ )] taking into account the effects of HRV. In fact, this formula has been evaluated in clinical studies and proved to be useful in distinguishing patients with high risk for sudden cardiac death in hypertrophic cardiomyopathy and patients with a history of ventricular fibrillation [11]. Although all studies did not always present data of the numerator (QTVN: 
QT variance/QTmean ${ }^{2}$ ) and denominator (HRVN: HR variance/HRmean ${ }^{2}$ ), increased QTVN rather than decreased HRVN might usually be the cause of QTVI to increase. Dobson suggested that evaluating both QTVN and HRVN is important in assessing the pathophysiology of QTVI. [6] As previously shown, HRVN changes with age; however, QTVN was not affected by age and remained stable without sex-related differences in children [7]. Thus, increase in QTVI might not necessarily indicate increase in QTVN, but could be due to decrease in HRVN. Actually, our analysis revealed a significant relationship between $\mathrm{LF} / \mathrm{HF}$ and $\mathrm{HF} /$ $(\mathrm{LF}+\mathrm{HF})$ with $\log _{10} \mathrm{HRVN}$; however, only weak relationship was observed with $\log _{10}$ QTVN. Therefore, it might be possible that increased sympathetic nerve activity reduces RR interval variability but has no marked effect on the QT interval variability. Schmidt $M$ and colleagues showed that QTV was increased in rapid eye movement sleep, reflective of high sympathetic drive and predicts death from cardiovascular disease [12]. However, HRV was not assessed in the paper. Likewise, augmented vagal nerve activity increases RR interval variability and could decrease QTVI, but its effect on QTVN is not clear. Further studies are required to evaluate the relationship between autonomic nerve balance and QTVN.

\section{Potential Clinical Implication}

Sudden Infant Death Syndrome (SIDS) is one of the leading causes of death in infants. Although the exact mechanisms contributing SIDS have not been fully elucidated until now, it is estimated that dysfunction of the autonomic nervous system regulation either respiratory or cardiovascular systems might play a part. The advantage of this study is that QTVI, which reflects myocardial repolarization variability, calculated from short-term ECG recording, can be used to assess the degree of autonomic tone. Thus, QTVI could be potentially used for predicting lethal arrhythmias, including SIDS. This should be explored in the future studies.

\section{Limitations}

This study has some limitations. First, this was a singlecenter study with a limited number of patients.

Second, we collected only one data in each subject. Thus, reproducibility of the parameters could not be assessed. Third, it may be true that sedatives affect the autonomic nervous activity and should be avoided to assess the autonomic balance, but in fact, it was impossible to ask the infant to rest on supine position for more than $2 \mathrm{~min}$. However, the amount of tricloryl syrup $(0.7 \mathrm{mg} / \mathrm{kg})$ used in this study is small so that we think effects of this medication on autonomic nervous activity is limited. Finally, we included only healthy infants, therefore it is unclear whether our results could be applied to infants with heart disease including hereditary channelopathies.

\section{Conclusion}

QTVI, which is calculated from non-invasive ECG and can detect abnormal myocardial repolarization instability, is significantly correlated with power spectral HRV parameters. QTVI reflects autonomic nerve balance in children.

Acknowledgements We express our gratitude to the Ministry of Health, Labor and Welfare of Japan for their funding to this study.

Funding This work was supported in part by the Japan Society for the Promotion of Science (KAKENHI) (\#26350944).

\section{Compliance with Ethical Standards}

Conflict of Interest The authors declare that they have no conflict of interest.

Ethical Approval All procedures performed in studies involving human participants were in accordance with the ethical standards of the institutional and/or national research committee and with the 1964 Helsinki declaration and its later amendments or comparable ethical standards.

Informed Consent Informed consent was obtained from all individual participants or parents/guardians included in the study.

Open Access This article is licensed under a Creative Commons Attribution 4.0 International License, which permits use, sharing, adaptation, distribution and reproduction in any medium or format, as long as you give appropriate credit to the original author(s) and the source, provide a link to the Creative Commons licence, and indicate if changes were made. The images or other third party material in this article are included in the article's Creative Commons licence, unless indicated otherwise in a credit line to the material. If material is not included in the article's Creative Commons licence and your intended use is not permitted by statutory regulation or exceeds the permitted use, you will need to obtain permission directly from the copyright holder. To view a copy of this licence, visit http://creativecommons.org/licenses/by/4.0/.

\section{References}

1. Atiga WL, Calkins H, Lawrence JH, Tomaselli GF, Smith JM, Berger RD (1998) Beat-to-beat Repolarization lability identifies patients at risk for sudden cardiac death. J Cardiovasc Electrophysiol 9:899-908. https://doi.org/10.1111/j.1540-8167.1998. tb00130.x

2. Berger RD, Kasper EK, Baughman KL, Marban E, Calkins H, Tomaselli GF (1997) Beat-to-beat QT interval variability: novel evidence for repolarization lability in ischemic and nonischemic dilated cardiomyopathy. Circulation 96:1557-1565. https://doi. org/10.1161/01.CIR.96.5.1557 
3. Massin MM, Maeyns K, Withofs N, Ravet F, Gérard P (2000) Circadian rhythm of heart rate and heart rate variability. Arch Dis Child 83:179-182. https://doi.org/10.1136/adc.83.2.179

4. Sassi R, Cerutti S, Lombardi F et al (2015) Advances in heart rate variability signal analysis: joint position statement by the e-Cardiology ESC Working Group and the European Heart Rhythm Association co-endorsed by the Asia Pacific Heart Rhythm Society. Europace 17:1341-1353. https://doi.org/10.1093/europace/ euv015

5. Baumert M, Porta A, Vos MA, Malik M, Couderc JP, Laguna P, Piccirillo G, Smith GL, Tereshchenko LG, Volders PG (2016) QT interval variability in body surface ECG: measurement, physiological basis, and clinical value: position statement and consensus guidance endorsed by the European Heart Rhythm Association jointly with the ESC Working Group on Cardiac Cellular Electrophysiology. Europace 18:925-944. https://doi.org/10.1093/europ ace/euv405

6. Dobson CP, Kim A, Haigney M (2013) QT variability index. Prog Cardiovasc Dis 56:186-194. https://doi.org/10.1016/j. pcad.2013.07.004

7. Kusuki H, Kuriki M, Horio K, Hosoi M, Matsuura H, Fujino M, Eryu Y, Miyata M, Yasuda T, Yamazaki T, Nagaoka S, Hata T (2011) Beat-to-beat QT interval variability in children: normal and physiologic data. J Electrocardiol 44:326-329. https://doi. org/10.1016/j.jelectrocard.2010.07.016

8. Takeuchi Y, Omeki Y, Horio K, Nishio M, Nagata R, Oikawa S, Mizutani Y, Nagatani A, Funamoto Y, Uchida H, Fujino M, Eryu Y, Boda H, Miyata M, Hata T (2017) Relationship between
QT and JT peak interval variability in prepubertal children. Ann Noninvasive Electrocardiol 22(4):1-6. https://doi.org/10.1111/ anec.12444 (Epub 2017 Feb 17)

9. Kojima A, Hata T, Sadanaga T, Mizutani Y, Uchida H, Kawai Y, Manabe M, Fujino M, Eryu Y, Boda H, Miyata M, Yoshikawa T (2018) Maturation of the QT variability index is impaired in preterm infants. Pediatr Cardiol 39(5):902-905. https://doi. org/10.1007/s00246-018-1839-2 (Epub 2018 Mar 12)

10. Heart rate variability. Standards of measurement, physiological interpretation, and clinical use. Task Force of the European Society of Cardiology and the North American Society of Pacing and Electrophysiology. (1996) Eur Heart. 17:354-81.

11. Atiga WL, Fananapazir L, McAreavey D, Calkins H, Berger RD (2000) Temporal repolarization lability in hypertrophic cardiomyopathy caused by beta-myosin heavy-chain gene mutations. Circulation 101:1237-1242. https://doi.org/10.1161/01. CIR.101.11.1237

12. Schmidt M, Baumert M, Penzel T, Malberg H, Zaunseder S (2019) Nocturnal ventricular repolarization lability predicts cardiovascular mortality in the Sleep Heart Health Study. Am J Physiol Heart Circ Physiol 316:H495-H505. https://doi.org/10.1152/ajphe art.00649.2018

Publisher's Note Springer Nature remains neutral with regard to jurisdictional claims in published maps and institutional affiliations. 